EPJ Web of Conferences 53, 04009 (2013)

DOI: $10.1051 /$ epjconf/20135304009

(C) Owned by the authors, published by EDP Sciences, 2013

\title{
Mass sensitive observables of the Pierre Auger Observatory
}

\author{
M. Unger ${ }^{1, a}$ for the Pierre Auger Collaboration ${ }^{2, b}$ \\ ${ }^{1}$ Karlsruher Institut für Technologie, Institut für Kernphysik, Germany \\ 2 Observatorio Pierre Auger, Av. San Martín Norte 304, 5613 Malargüe, Argentina
}

\begin{abstract}
In this article we will discuss measurements of the longitudinal development of air showers at the Pierre Auger Observatory. The longitudinal development of the electromagnetic component can be directly observed by the fluorescence telescopes of the Auger Observatory and we will present the results on the evolution of the average shower maximum and its fluctuations as a function of energy. Moreover, two observables from the surface detector, the asymmetry of the rise time of the station signals and the muon production depth, will be discussed and the measurements will be compared to predictions from air shower simulations for different primary particle types.
\end{abstract}

\section{INTRODUCTION}

The measurement of the mass composition of ultra-high energy cosmic rays is important to understand their astrophysical origin. However, since ultra-high energy primary particles can only be measured indirectly via extensive air showers, the interpretation of experimental data in terms of mass composition is difficult due to our lack of precise knowledge of the properties of hadronic interactions at these energies (see e.g. [1] for a recent review on mass composition estimates using air showers). To estimate the uncertainties of air shower modeling one can compare the data to predictions from simulations using different hadronic interaction models. But since it is not guaranteed that current models cover the full phase space of possible characteristics of interactions at ultra-high energies, it is advisable to study different mass sensitive observables that probe different aspects of air shower cascades.

In this article we present four measurements of air shower characteristics related to the primary mass composition using data of the Pierre Auger Observatory [2]. The hybrid nature of the Observatory allows for studies of the longitudinal development of the electromagnetic component of air showers as well as the hadronic component via muons detected at ground level. The Observatory consists of a surface detector (SD) and a fluorescence detector (FD). An area of $3000 \mathrm{~km}^{2}$ is covered by an array of over 1600 water-Cherenkov detectors arranged on a triangular grid with a spacing of $1.5 \mathrm{~km} \mathrm{[3].}$ The array is overlooked by 27 fluorescence telescopes [4] which observe the longitudinal air shower development during clear new- to half-moon nights with a duty cycle of about $15 \%$.

The layout of the Observatory along with an example of a measured ultra-high energy shower is shown in Fig. 1. As can be seen, this particular event with a primary energy of $(47 \pm 1) \mathrm{EeV}$ triggered 16 surface detector stations and all four FD sites including the recently installed high elevation telescopes [5]. The simultaneous measurement of ultra-high energy showers by several fluorescence telescopes not only improves the quality of the reconstructed air shower observables, but the redundant measurements allow for systematic studies of our understanding of the detectors and the atmosphere.

ae-mail: Michael. Unger@kit.edu

${ }^{b}$ For the full authorlist see Appendix "'Collaborations" in this volume.

This is an Open Access article distributed under the terms of the Creative Commons Attribution License 2.0, which permits unrestricted use, distribution, and reproduction in any medium, provided the original work is properly cited. 


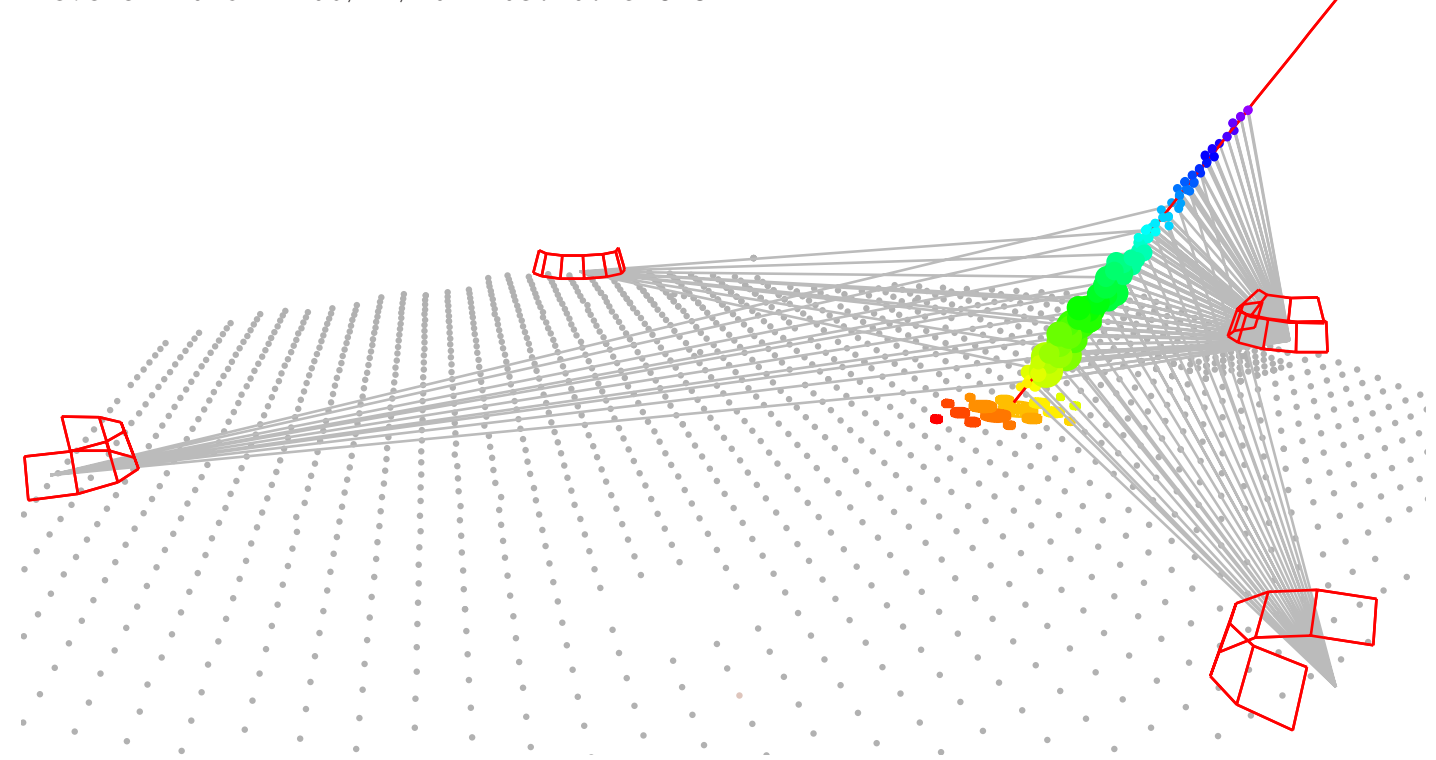

Figure 1. Example of an ultra-high energy event measured by the Pierre Auger Observatory with the surface detector and the four fluorescence stations including the new HEAT telescopes. Colors denote the times at the shower and ground respectively. The size of the markers correspond to the magnitude of measured signals at ground and reconstructed energy deposits at the shower. Red squares illustrate the field of view of the 27 fluorescence telescopes, the viewing directions of triggered pixels are shown as gray lines and the surface detector stations are displayed as gray dots. The averaged energies and shower maxima from the four fluorescence sites yield $E=(4.7 \pm 0.1) \times 10^{19} \mathrm{eV}$ and $X_{\max }=(730 \pm 3) \mathrm{g} / \mathrm{cm}^{2}$ respectively.

Furthermore, the coincident measurement of air showers with the fluorescence and surface detector is used, among other things, for a model independent calibration of the energy scale of the surface detector [6] and to achieve a very precise reconstruction of the FD-only shower geometries [7].

\section{FLUORESCENCE DETECTOR DATA}

One of the most robust mass sensitive observables is given by the shower maximum, $X_{\max }$, which is the position at which an air-shower deposits the maximum energy per unit of atmospheric slant depth. This observable can be directly measured by fluorescence telescopes. Light primaries are expected to penetrate on average deeper in the atmosphere than heavy primaries. Moreover, due to their larger cross section and larger number of nucleons, heavy nuclei are expected to exhibit less event-by-event fluctuations concerning $X_{\max }$ than light primaries.

The measured energy evolution of the average shower maximum, $\left\langle X_{\max }\right\rangle$ is shown in the left panel of Fig. $2[8,9]$. In total 6744 events were selected after application of quality and fiducial cuts, see [10] for a detailed description of the data analysis. The systematic uncertainty in $\left\langle X_{\max }\right\rangle$ ranges from $10 \mathrm{~g} / \mathrm{cm}^{2}$ at low energy to $13 \mathrm{~g} / \mathrm{cm}^{2}$ at high energy and includes contributions from the uncertainties in the calibration, the atmospheric monitoring data $[11,12]$, the reconstruction and the event selection.

The elongation rate $D_{10}=\mathrm{d}\left\langle X_{\max }\right\rangle \mathrm{d} \lg E$ is a measure of the rate of change of the average shower maximum per decade of energy [13] and can be used to characterize the change of the average logarithmic mass of the primaries, since $D_{10} \propto \mathrm{d}\langle\ln A\rangle / \mathrm{d} \lg E$. A fit to the $\left\langle X_{\max }\right\rangle$ measurements using only one elongation rate does not describe our data well $\left(\chi^{2} / \mathrm{Ndf}=54 / 11\right)$. Allowing for a break in the elongation rate we find $82_{-8}^{+48} \mathrm{~g} / \mathrm{cm}^{2} /$ decade below $\lg (E / \mathrm{eV})=18.38_{-0.17}^{+0.07}$ and $27_{-8}^{+3} \mathrm{~g} / \mathrm{cm}^{2} / \mathrm{decade}$ 

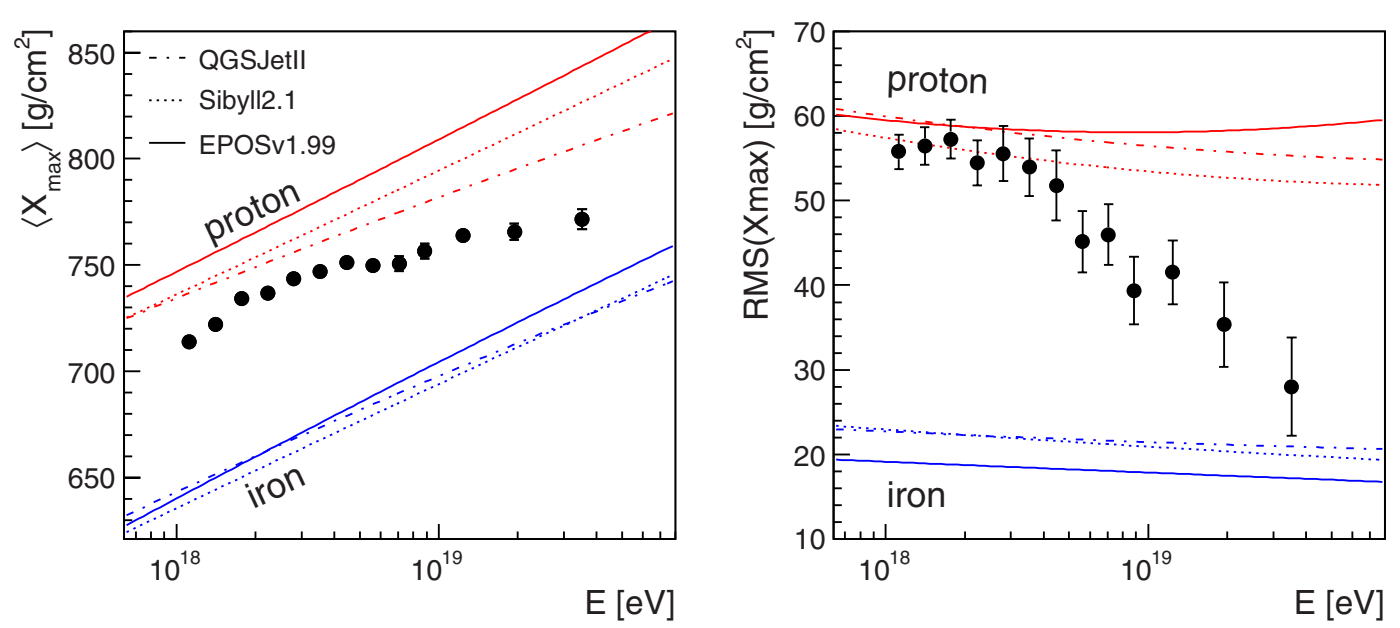

Figure 2. Energy evolution of the average of the shower maximum (left) and its fluctuation (right) compared to air shower simulations [22, 24-26].

above that energy with a $\chi^{2} / \mathrm{Ndf}=7.4 / 9$. The low energy value is close to the bound given by the elongation rate theorem which states that the change of $\left\langle X_{\max }\right\rangle$ per decade cannot exceed the radiation length in air $\left(X_{0} \cdot \ln 10=84.3 \mathrm{~g} / \mathrm{cm}^{2}\right)$ unless the composition is changing from heavy to light primaries. On the other hand, the comparison of the measured elongation rate at high energies to air shower predictions for a constant composition $\left(D_{10}^{\text {const }} \approx 50-60 \mathrm{~g} / \mathrm{cm}^{2} /\right.$ decade) would suggest a transition from lighter to heavier primaries. Similar conclusions hold for the comparison of the absolute $\left\langle X_{\max }\right\rangle$ values to the air shower predictions shown as lines in Fig. 2.

The standard deviation of the $X_{\max }$ distributions, $\operatorname{RMS}\left(X_{\max }\right)$, is shown in the right panel of Fig. 2. It has been corrected for the experimental $X_{\max }$ resolution that ranges from $27 \mathrm{~g} / \mathrm{cm}^{2}$ at low energy to $18 \mathrm{~g} / \mathrm{cm}^{2}$ at high energy by subtraction in quadrature. The systematic uncertainty on $\operatorname{RMS}\left(X_{\max }\right)$ is at the level of $5 \mathrm{~g} / \mathrm{cm}^{2}$. As can be seen, $\operatorname{RMS}\left(X_{\max }\right)$ decreases gradually with energy from $55 \mathrm{~g} / \mathrm{cm}^{2}$ to $26 \mathrm{~g} / \mathrm{cm}^{2}$ from at around the same energy where the elongation rate changes its value. For some of the air shower predictions, the data would have to be adjusted within their systematic uncertainties to simultaneously match both $\left\langle X_{\max }\right\rangle$ and $\operatorname{RMS}\left(X_{\max }\right)$ for a given composition mixture $\left(\left\langle X_{\max }\right\rangle\right.$ downward and $\operatorname{RMS}\left(X_{\max }\right)$ upward and/or the energy scale upward). It is worthwhile noting that this 'tension' between the Auger data and the hadronic interaction models is expected to diminish when the models will have been tuned to the recent LHC data [14].

Both, $\left\langle X_{\max }\right\rangle$ and $\operatorname{RMS}\left(X_{\max }\right)$, have been obtained from the full $X_{\max }$ distributions and examples of these are shown in the upper panel of Fig. 3 at four energies. The predictions for three simple composition scenarios are superimposed: pure proton composition, pure iron composition and a 50:50 mixture of proton and iron. Neither of these simple models can describe the data perfectly, but at low energies the distributions are closest to the proton simulation. Air shower predictions are more uncertain for the $\left\langle X_{\max }\right\rangle$ than for the fluctuations due to the additional dependence of $\left\langle X_{\max }\right\rangle$ on the multiplicity in hadronic interactions [15] and because a large contribution to the fluctuations comes from the hadronic interaction length for which the model predictions were recently validated at $\mathrm{EeV}$ energies [16]. We therefore compare the shape of the distributions, $X_{\max }-\left\langle X_{\max }\right\rangle$, to the simulations in the lower panel of Fig. 3. As can be seen, in this representation the various models predict a nearly universal shape for a given composition mixture. At low energy, the shape of the data is compatible with a very light or mixed composition, whereas at high energies, the narrow shape would favor a significant fraction of nuclei (CNO or heavier). 

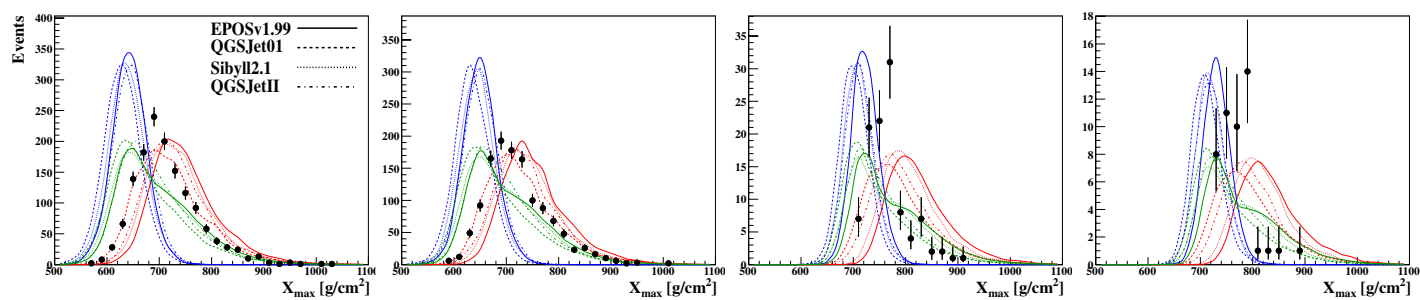

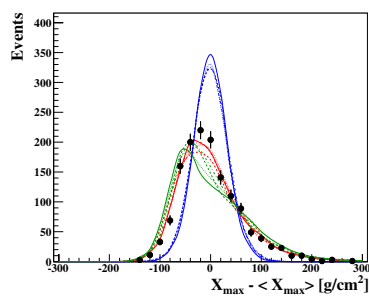

(a) $18.0<\lg E / \mathrm{eV}<18.1$

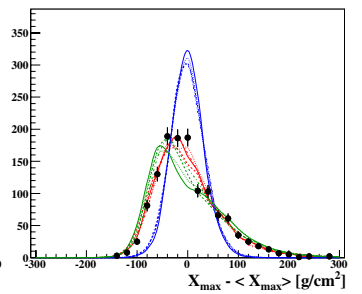

(b) $18.1<\lg E / \mathrm{eV}<18.2$

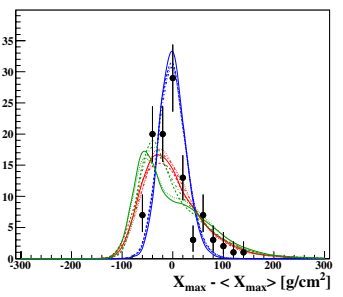

(c) $19.2<\lg E / \mathrm{eV}<19.4$

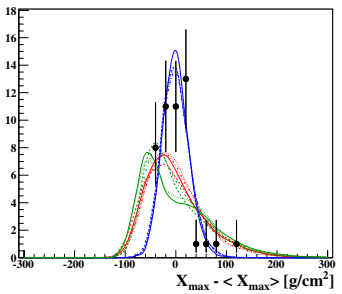

(d) $\lg E / \mathrm{eV}>19.4$

Figure 3. Measured $X_{\max }$ distributions at different energies (black dots). Top panel: absolute $X_{\max }$ scale, bottom panel: shape of distribution relative to $\left\langle X_{\max }\right\rangle$. Predictions from air shower simulations are normalized to the number of measured events and smeared with the detector resolution. Iron simulations are shown in blue, proton simulations in red and a 50:50 proton/iron mixture in green.

\section{SURFACE DETECTOR DATA}

In principle, the number of muons in an air shower is an observable that is well suited for mass composition studies, because heavy primaries produce more muons than lighter ones. However, e.g. the combined analysis of fluorescence and surface detector data from the Pierre Auger Observatory shows that air shower simulations produce too few muons [17] and therefore for the surface detector analysis we currently focus on observables that are sensitive to the longitudinal air shower development and robust with respect to the absolute number of muons in air showers.

In the rise-time asymmetry analysis, we exploit the fact that due to the multiple scattering of electrons in air the electromagnetic component of an air shower is more spread in time than its muonic component. Therefore the rise-time of the surface detector signals, i.e. the time it takes to go from $10 \%$ to $50 \%$ of the total integrated signal, can be used as a proxy for the muon-to-electron ratio in a surface detector station. For non-vertical showers, the rise-time can be measured at different longitudinal depths by comparing signals in stations located up- and down-stream of the incoming shower direction. The zenith angle at which the corresponding rise-time asymmetry is maximal is then used as an estimator for the average longitudinal development of air showers [18]. The energy evolution of this estimator, $\sec (\theta)_{\max }$, is shown in the left panel of Fig. 4. This measurement is based on 18581 surface detector events and has a systematic uncertainty that amounts to $\lesssim 10 \%$ of the predicted proton-iron difference. The comparison of $\sec (\theta)_{\max }$ to air shower simulations leads to a similar interpretation as in case of $\left\langle X_{\max }\right\rangle$, namely a predominantly light composition at low energies and a gradual increase of the average mass of primary cosmic rays towards high energies.

Another mass-sensitive observable currently studied with the surface detector is related to the production depths of muons. These can be inferred from a geometric back-projection [20] of the time structure of the signals recorded in the water Cherenkov stations to the shower axis. Only showers with zenith angles between $55^{\circ}$ and $65^{\circ}$ and stations beyond $1800 \mathrm{~m}$ distance to the core are used to obtain a good resolution of the production depth and to avoid a contamination from the electromagnetic air shower component. In this way, the longitudinal distribution of muon production depths can be 

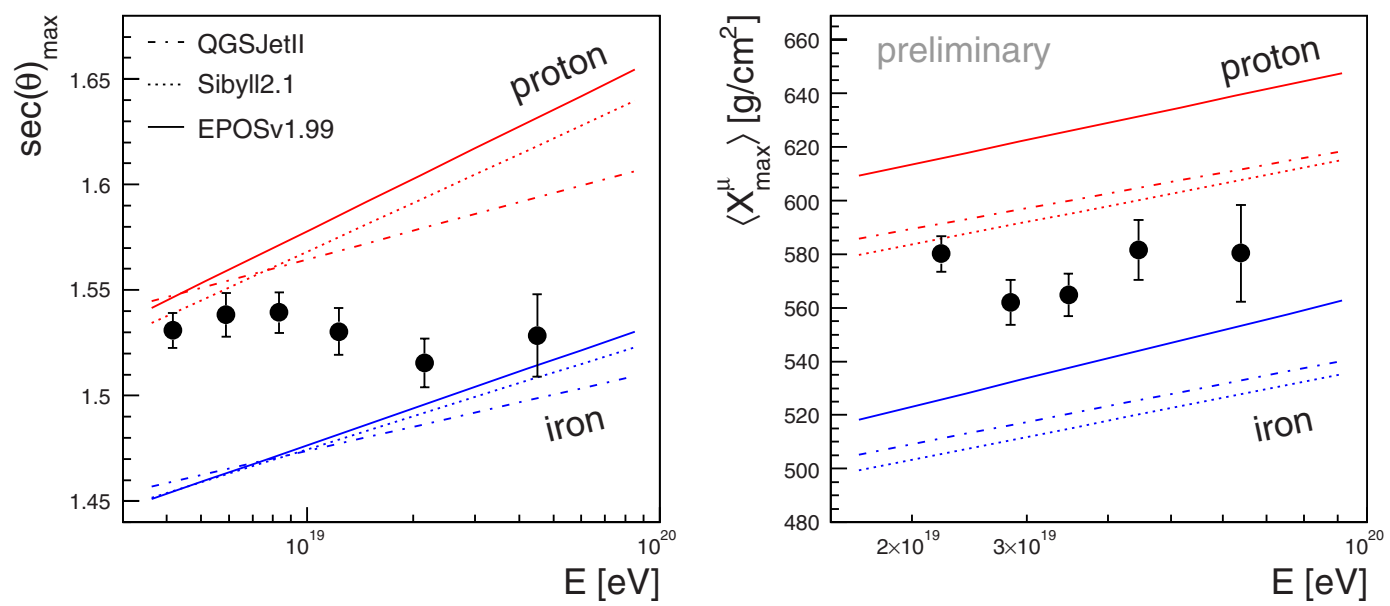

Figure 4. Energy evolution of zenith angle at which the rise-time asymmetry is maximal (left) and the average of the depth of the maximum of the muon production depth distributions (right) compared to air shower simulations [23-26].

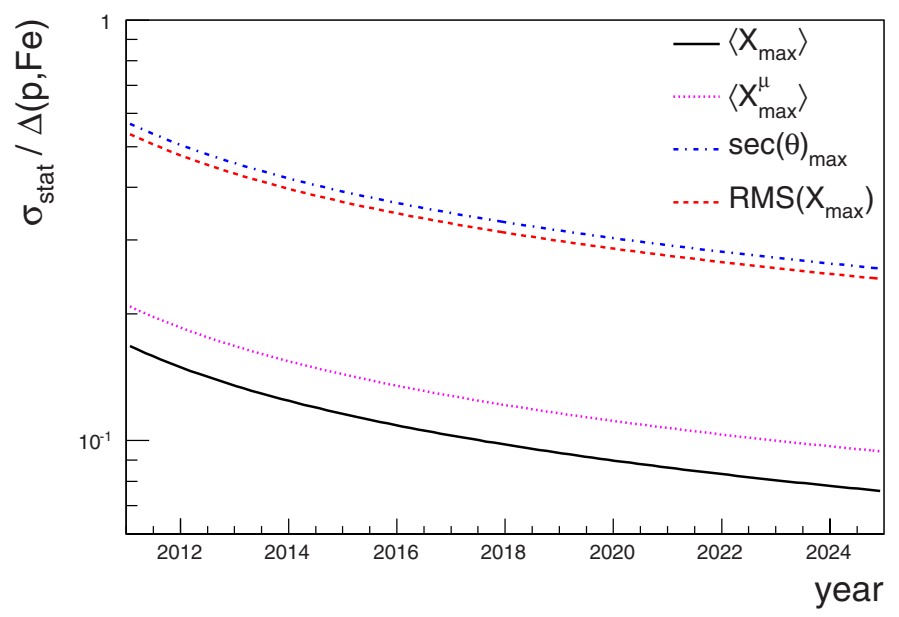

Figure 5. Expected time evolution of the statistical uncertainty, $\sigma_{\text {stat }}$ of the four composition sensitive observables discussed in this article above $56 \mathrm{EeV}$ in units of the proton-iron difference, $\Delta(\mathrm{p}, \mathrm{Fe})$.

measured on an event-by-event basis and the depth of the maximum of the distribution, $X_{\max }^{\mu}$, can be determined [21]. The energy evolution of $\left\langle X_{\max }^{\mu}\right\rangle$ is shown in the right panel of Fig. 4 based on 290 events above $20 \mathrm{EeV}$ with a systematic uncertainty of $14 \mathrm{~g} / \mathrm{cm}^{2}$. As can be seen, the data points are between the predictions for proton and iron primaries. For SIBYLL2. I and QGSJETII a rather light composition mixture would follow whereas the positions of the EPOSI.99 lines relative to the data are in better agreement with the composition interpretation of the $\left\langle X_{\max }\right\rangle$ and $\sec (\theta)_{\max }$ data. This clearly demonstrates the advantages of using independent variables for mass composition studies. In addition to a reduction of experimental systematics, the comparison of fluorescence and surface detector data to air shower simulations allows to reduce the model-dependence of composition estimates and a necessary condition for the use of particular hadronic interaction model is that it should lead to a consistent mass composition interpretation for all experimental observables. However, within the present uncertainties of our measurements none of the three models shown in Figs. 2 and 4 can be excluded yet. 
EPJ Web of Conferences

\section{OUTLOOK}

The main topic of this workshop was the 'Future Directions in UHECR Physics'. One of the major challenges of the next decade will be the measurement of the cosmic ray composition at ultra-high energies to establish the nature of the observed flux suppression and to help to select events with small mass (and therefore charge) for anisotropy studies. The expected time evolution of the statistical uncertainty in units of the expected proton-iron separation above $56 \mathrm{EeV}$ for the four mass sensitive observables that were discussed in this article is shown in Fig. 5. As can be seen, even after ten years of further data taking with the Pierre Auger Observatory, the uncertainties are expected to be at the $10 \%$ level using either of our currently most powerful observables, $\left\langle X_{\max }\right\rangle$ or $\left\langle X_{\max }^{\mu}\right\rangle$. Whereas such precision would allow us to constrain possible composition scenarios at ultra-high energies, it is not sufficient for a detailed study of the composition beyond an average logarithmic mass and surely not enough for an event-by-event charge separation. Besides the further improvement of the data analysis (e.g. by using multivariate techniques), a much larger exposure and/or improved instrumentation seems to be mandatory for composition studies at ultra-high energies.

\section{References}

[1] K.H. Kampert and M. Unger, Astropart. Phys. 35 (2012) 660

[2] The Pierre Auger Collaboration, Nucl. Instrum. Meth. A 523 (2004) 50

[3] The Pierre Auger Collaboration, Nucl. Instrum. Meth. A 586 (2008) 409

[4] The Pierre Auger Collaboration, Nucl. Instrum. Meth. A 620 (2010) 227

[5] H.J. Mathes for the Pierre Auger Collaboration, Proc. 32nd ICRC 3 (2011) 149, arXiv: $1107.4807 \mathrm{v} 1$

[6] I.C. Mariş for the Pierre Auger Collaboration, these proceedings

[7] P. Sommers, Astropart. Phys. 3 (1995) 349; B. Dawson et al., Astropart. Phys. 5 (1996) 239

[8] The Pierre Auger Collaboration, Phys. Rev. Lett. 104 (2010) 091101

[9] P. Facal for the Pierre Auger Collaboration, Proc. 32nd ICRC 2 (2011) 105, arXiv:1107.4804v1

[10] V. de Souza for the Pierre Auger Collaboration, these proceedings

[11] The Pierre Auger Collaboration, Astropart. Phys. 35 (2012) 591

[12] The Pierre Auger Collaboration, Astropart. Phys. 33 (2010) 108

[13] J. Linsley, Proc. 15th ICRC 112 (1977) 89; T. K. Gaisser, T. J. K. McComb, K. E. Turver, Proc. 16th ICRC 9 (1979) 258; J. Linsley, A. Watson, Phys. Rev. Lett. 46 (1981) 459

[14] T. Pierog et al., these proceedings

[15] R. Ulrich et al., Phys. Rev. D 83 (2011) 054026

[16] R. Ulrich for the Pierre Auger Collaboration, these proceedings

[17] A. Yushkov for the Pierre Auger Collaboration, these proceedings

[18] M. Dova et al., Astropart. Phys. 31 (4) (2009) 312

[19] D. Garcia-Pinto et al. [Pierre Auger Coll.], Proc. 32nd ICRC 2 (2011) 87, arXiv:1107.4804v1

[20] L. Cazon et al., Astropart. Phys. 21 (2004) 71

[21] D. García-Gámez for the Pierre Auger Collaboration, these proceedings

[22] T. Bergmann et al., Astropart. Phys. 26 (2007) 420

[23] D. Heck et al., Forschungszentrum Karlsruhe FZKA 6019 (1998)

[24] T. Pierog, K. Werner, Phys. Rev. Lett. 101 (2008) 171101

[25] S. Ostapchenko, Phys. Rev. D 83 (2011) 014018

[26] E. Ahn et al., Phys. Rev. D 80 (2009) 094003 